OPEN ACCESS

Edited by:

Lidia Santarpia,

University of Naples Federico II, Italy

Reviewed by:

Amir Hadi

Isfahan University of Medical

Sciences, Iran

Meghit Boumediene Khaled,

University of Sidi-Bel-Abbès, Algeria

*Correspondence:

Yi Lei

leiyi111@scu.edu.cn

Yong-Gang Zhang

jebm_zhang@yahoo.com

tThese authors have contributed equally to this work and share first authorship

Specialty section:

This article was submitted to

Clinical Nutrition

a section of the journal

Frontiers in Nutrition

Received: 14 June 2021

Accepted: 12 August 2021

Published: 07 September 2021

Citation:

Guo R, Li N, Yang R, Liao X-Y, Zhang Y, Zhu B-F, Zhao Q, Chen L, Zhang Y-G and Lei $Y$ (2021) Effects of the Modified DASH Diet on Adults

With Elevated Blood Pressure or Hypertension: A Systematic Review and Meta-Analysis.

Front. Nutr. 8:725020

doi: 10.3389/fnut.2021.725020

\section{Effects of the Modified DASH Diet on Adults With Elevated Blood Pressure or Hypertension: A Systematic Review and Meta-Analysis}

\begin{abstract}
Ru Guo ${ }^{1 t}$, Nian Li ${ }^{2 t}$, Rong Yang ${ }^{1}$, Xiao-Yang Liao ${ }^{1}$, Yu Zhang ${ }^{1}$, Ben-Fu Zhu ${ }^{1}$, Qian Zhao ${ }^{1}$, Lingmin Chen ${ }^{3}$, Yong-Gang Zhang ${ }^{4,5 *}$ and Yi Lei ${ }^{1 *}$

${ }^{1}$ International Medical Center/Department of General Practice and National Clinical Research Center for Geriatrics, West China Hospital, Sichuan University, Chengdu, China, ${ }^{2}$ Department of Medical Administration, West China Hospital, Sichuan University, Chengdu, China, ${ }^{3}$ Department of Anesthesiology and National Clinical Research Center for Geriatrics, West China Hospital, Sichuan University and The Research Units of West China, Chinese Academy of Medical Sciences, Chengdu, China, ${ }^{4}$ Department of Periodical Press and National Clinical Research Center for Geriatrics, West China Hospital, Sichuan University, Chengdu, China, ${ }^{5}$ Chinese Evidence-Based Medicine Center, West China Hospital, Sichuan University, Chengdu, China
\end{abstract}

Background: The modified Dietary Approaches to Stop Hypertension (DASH) diet was a potentially effective treatment for pre-hypertensive and hypertensive patients. The evidence for the effect of the modified DASH diet on blood pressure reduction was inconsistent. The study was designed to assess the effects of the modified DASH diet on blood pressure (BP) in hypertensive and pre-hypertensive adults.

Methods: We searched Medline, Embase, CENTRAL, CNKI, VIP, Wanfang Data, SINOMED, Google Scholar, the World Health Organization's International Clinical Trials Registry Platform, and Clinicaltrials.gov from inception to July 1st, 2021. Randomized controlled trials (RCTs) assessing the effects of the modified DASH diet on systolic and diastolic BP, cardiovascular risk factors (body weight, body mass index, waist circumference, fasting glucose, blood lipids), cardiovascular events, and all-cause mortality were included. Statistical analysis was performed using Stata software. Risk of bias was assessed with the Cochrane tool and quality of evidence with GRADE.

Results: A total of 10 RCTs were included. Compared with control diet, the modified DASH diet could reduce mean systolic $(-3.26 \mathrm{mmHg}$; 95\% confidence interval -5.58 , $-0.94 \mathrm{mmHg} ; P=0.006)$ and diastolic $(-2.07 \mathrm{mmHg}$; $95 \%$ confidence interval -3.68 , $-0.46 \mathrm{mmHg} ; P=0.01) \mathrm{BP}$. Compared with the controlling diet, the modified DASH diet could reduce systolic BP to a greater extent in trials with a mean baseline BP $\geq 140 / 90$ $\mathrm{mmHg}$ compared with $<140 / 90 \mathrm{mmHg}$. Diastolic BP reduction was greater when the mean body mass index was $\geq 30 \mathrm{~kg} / \mathrm{m} 2$ than $<30 \mathrm{~kg} / \mathrm{m}^{2}$. Diastolic BP reduction was more marked in trials with a follow-up time of $>8$ weeks compared with $\leq 8$ weeks. The modified DASH diet could affect mean waist circumference (difference: $1.57 \mathrm{~cm}$; $95 \%$ confidence interval $-2.98,-0.15$ ) and triglyceride concentration (difference: $1.04 \mathrm{~mol} / \mathrm{L}$; $95 \%$ confidence interval $-1.47,-0.60)$. 
Conclusions: The modified DASH diet can reduce BP, waist circumference, and triglyceride concentration in hypertension patients. A higher baseline BP is associated with more marked systolic and diastolic BP reduction.

Systematic Review Registration: PROSPERO registration number:
CRD42020190860.

Keywords: dietary approaches to stop hypertension, hypertension, randomized controlled trial, meta-analysis, systematic review

\section{INTRODUCTION}

Hypertension, a primary risk factor for cardiovascular disease, is the leading cause of premature death worldwide and accounts for $\sim 12.8 \%$ of global deaths (1-3). Systolic BP (SBP) or diastolic BP (DBP) increased by $20 / 10 \mathrm{mmHg}$ will increase the risk of cardiovascular diseases by two times $(4,5)$. However, $<20 \%$ of patients with hypertension achieve effective BP control (1). Therefore, lifestyle modifications, including a healthy diet, are recommended as potential therapeutic strategies (6-8).

The Dietary Approaches to Stop Hypertension (DASH) diet was the most typical dietary treatment strategy for BP control. It was abundant in fruits, vegetables and low-fat dairy products, with more fish, nuts and legumes, and a moderate sodium restriction (9). It reduced SBP and DBP by $5.5 \mathrm{mmHg}$ and 3.0 $\mathrm{mmHg}$ in the first DASH clinical trial 20 years ago (10). DASH diet was effective in the reduction of BP and other cardiovascular risk factors, including blood glucose, blood lipids, body weight, and waist circumference (10-13). Hence, it was recommended in the treatment of hypertension (10-13). However, poor adherence was the main barrier, and only a small population fully adhered to the diet (3). Inappropriate translation, low spreading velocity, difficulty to follow, and contradiction with dietary culture were the main reasons (14-17).

The modified DASH diet, which expanded or reduced nutrient composition based on the original DASH diet, was one solution to poor adherence. It reduced the sodium level of DASH diet $(6-3 \mathrm{~g})$ to enhance the antihypertensive effect in DASH-Sodium research, which was the first study to modify the DASH diet (7). Many studies have been performed to assess the efficacy of modified DASH for hypertension patients (18-20). However, the results were inconsistent. Some studies reported that the modified DASH diet could reduce BP (18-20), body mass index (BMI) (19), blood sugar (18), and lipidemia (21); while other studies suggested the modified DASH diet might increase BP (22), blood sugar (18), and insulin resistance (23). Thus, we conducted a systematic review and meta-analysis to assess the efficacy of modified DASH diet for hypertension patients.

\footnotetext{
Abbreviations: DASH, The Dietary Approaches to Stop Hypertension; BP, blood pressure; RCT, randomized controlled trial; SBP, Systolic blood pressure; DBP, diastolic blood pressure; ISH, International Society of Hypertension; BMI, body mass index; PRISMA, Preferred Reporting Items for Systematic Reviews and Meta-Analyses; MDs, mean differences; SMDs, standardized mean differences; TG, Triglycerides; TC, Total cholesterol; LDL, Low-density lipoprotein; HDL, High-density lipoprotein.
}

\section{METHODS}

Study Registration and Reporting Guideline The protocol was registered on PROSPERO (CRD4201007296). It was reported according to Preferred Reporting Items for Systematic Reviews and Meta-Analyses (PRISMA) guidelines (Supplemental Material 1) (24).

\section{Inclusion Criteria and Exclusion Criteria}

The inclusion criteria were as follows: (1) study design: published and ongoing randomized controlled trials(RCTs); (2) participants: adults $\geq 18$ years with mean SBP and DBP $\geq 130$ or $\geq 80 \mathrm{mmHg}$; (3) intervention: advised or administered any type of modified DASH diet, either as a sole intervention or in combination with other interventions (e.g., exercise), and interventions described as "DASH-Sodium," "DASH-Plus," or "WELL diet" were included when they modified species or weight with foods in the DASH diet; (4) comparison: regular diet, traditional diet, typical American diet, or any other diet not related to DASH; (5) outcomes: primary outcomes included SBP and DBP, and secondary outcomes included cardiovascular risk factors [body weight, BMI, waist circumference, fasting glucose, total cholesterol (TC), triglycerides (TG), low-density lipoprotein (LDL), high-density lipoprotein (HDL)], cardiovascular events, and all-cause mortality. Searches were limited to the English and Chinese languages. Exclusion criteria were as follows: (1) study design: case reports, case series, observational studies, and animal research; (2) participants: individuals $<18$ years of age, pregnant women, and acute patients; (3) comparison: other different routes of modified DASH diet; (4) outcomes: outcomes with insufficient data, such as BP data at baseline or endpoint only.

\section{Databases and Search Strategy}

We conducted a systematic search of several databases, including Medline (Ovid), EMbase (Ovid), Cochrane Central Register of Controlled Trials, CNKI, VIP, WanFang Data and SINOMED. The initially search was performed on October, 2020, and the updated search was performed on July 1st, 2021. We also searched Google Scholar, ClinicalTrials.gov, and the WHO's International Clinical Trials Registry Platform to identify ongoing trials. The following search terms were used: (DASH diet OR dietary approaches to stop hypertension) AND (hypertension OR blood pressure OR high blood pressure OR increased blood pressure OR elevated blood pressure) (Supplemental Material 2). Additionally, to identify relevant studies, we manually screened references in the included studies. 


\section{Study Selection}

Two researchers independently screened the titles and abstracts of records and performed a full-text review to evaluate all potentially eligible studies. Disagreements were resolved by discussion with a third reviewer. The following data were extracted from each study: (i) author, country, language, and year of publication; (ii) methods (study design, study analysis, sample sizes for both interventions and controls); (iii) risk of bias assessment; (iv) sample characteristics for both interventions and controls separately (gender ratio, mean age, ethnic background, mean baseline BP, mean BMI, specific measures and sodium intake of interventions and controls, methods of modified diet, follow-up duration, hypertension prevalence, anti-hypertension treatment, energy restriction of interventions). If the same dataset had been published more than once, we included the study with the largest number of participants or the best complete findings.

\section{Data Extraction}

Two researchers independently extracted data from the included studies using a standard data extraction form. Disagreements were resolved by consensus or by a discussion with a third reviewer.

\section{Assessment of the Risk of Bias in Included Studies}

Two researchers independently assessed the risk of bias of all included trials using the Cochrane risk bias tool (25). Quality of evidence were analyzed by using the grading of recommendations assessment, development, and evaluation (GRADE) approach.

\section{Statistical Analysis}

The meta-analysis was conducted using Revman 5.3 software (26) and Stata software (version 12.0; StataCorp, College Station, TX). Mean differences (MDs) or standardized MDs (SMDs) before and after the intervention was obtained. Cochran's Q statistic (significance level, $P<0.10$ ) and the $I^{2}$ statistic were calculated to assess heterogeneity. We defined an $I^{2}$ value of $<50 \%$ as low heterogeneity and an $I^{2}$ value $>50 \%$ as high heterogeneity. A fixed-effects model was used when there was no significant heterogeneity; otherwise, the random-effects model was used. Sensitivity analysis was performed to analyze the stability of the results by sequentially omitting one study. When there were enough studies on BP outcomes, subgroup and meta-regression analyses were conducted to investigate the differential effects of the modified DASH diet on hypertension and to explore heterogeneity. These factors included age, gender, baseline BP $(<140 / 90$ or $\geq 140 / 90 \mathrm{mmHg})$, BMI $\left(<30\right.$ or $\left.\geq 30 \mathrm{~kg} / \mathrm{m}^{2}\right)$, duration of follow up ( $\leq 8$ or $>8$ weeks), sodium intake $(\leq 1,500$ or $>1,500 \mathrm{mg} / \mathrm{d}$ ), control measures (healthy diet or common diet). Publication bias was assessed using visual inspection of funnel plots. A $P<0.05$ was considered statistically significant.

\section{RESULTS}

\section{Eligible Studies and Study Characteristics}

We initially identified 5,210 results through the literature search, with four studies identified through manual screening of reference lists from the included studies. After removing duplicates, 4,455 titles and abstracts were screened, and 267 articles remained for full-text review. Finally, a total of 10 studies were included (Figure 1) (7, 16, 18-20, 22, 27-30).

The studies included 2,416 participants with an age range from 45.10 to 62.20 years with a BP of $127 / 81$ to $165 / 85 \mathrm{mmHg}$. Four studies were conducted in the USA and the others were from China, South Korea, Canada, Pakistan, and Brazil. Table 1 shows the characteristics of the included studies. More details are shown in Supplemental Materials 3, 4. The results of the risk bias assessment are shown in Figure 2 and Supplemental Material 5.

\section{Effect on BP}

All ten trials reported BP before and after the intervention. Compared with the control diet, the modified DASH diet was associated with a significant reduction in SBP (MD: -3.26 $\mathrm{mmHg}$; 95\% CI -5.58, -0.94) and DBP (MD: $-2.07 \mathrm{mmHg}$; 95\% CI -3.68, -0.46) (Figures 3, 4). Subgroup analysis showed that the reduction in SBP and DBP was significantly greater in subgroups with baseline SBP/DBP values of $\geq 140 / 90 \mathrm{mmHg}$ compared with $<140 / 90 \mathrm{mmHg}$ (SBP: $P=0.02$; DBP: $P=$ $0.01)$. Furthermore, DBP reduction was significantly greater in the subgroup with a longer follow-up period compared with a shorter follow-up period $(P=0.04)$. Additionally, DBP reduction was significantly greater in subgroup with a BMI $\geq 30 \mathrm{~kg} / \mathrm{m}^{2}$ compared with $<30 \mathrm{~kg} / \mathrm{m}^{2}(P=0.04)$. SBP and DBP reductions showed no statistic difference between subgroups aged 45-60 years and subgroups aged 60-74 years (SBP: $P=0.25$; DBP: $P=0.26)$. No statistical difference was observed in subgroups with a sodium intake of $>2,400 \mathrm{mg} / \mathrm{d}$ vs. $\leq 2,400 \mathrm{mg} / \mathrm{d}$ (SBP: $P$ $=0.13$; DBP: $P=0.26$ ). No statistical difference was observed in subgroups with healthy diet vs. common diet (SBP: $P=$ 0.40 ; DBP: $P=0.20$ ). We found no significant interaction in prespecified subgroup meta-regressions for age, gender, baseline BP, BMI, duration of follow up, and sodium intake. However, control measures (healthy diet or common diet) appeared to be the main source of heterogeneity (SBP: $P$ for interaction $=0.039$; DBP: $P$ for interaction $=0.048)($ Supplemental Materials 6, 7).

\section{Effect on Cardiovascular Risk Factors}

As to cardiovascular risk factors, the waist data from two RCTs showed that mean waist was $1.57 \mathrm{~cm}$ lower in people receiving modified DASH diet than in those receiving control diet (22, 29). Besides, two studies showed that the reduction in TG concentration was greater in the modified DASH diet group compared with the control group (MD: $-1.04 \mathrm{mmol} / \mathrm{L})(19,29)$. However, another two studies showed no significant difference in body weight reduction between the modified DASH diet group and the control group (MD: $0.31 \mathrm{~kg})(19,22)$. BMI was also not significantly reduced (MD: $0.6 \pm 2.55$ ). Additionally, no reduction in TC (MD: $-0.98 \mathrm{mmol} / \mathrm{L})$, LDL (MD: -0.41 


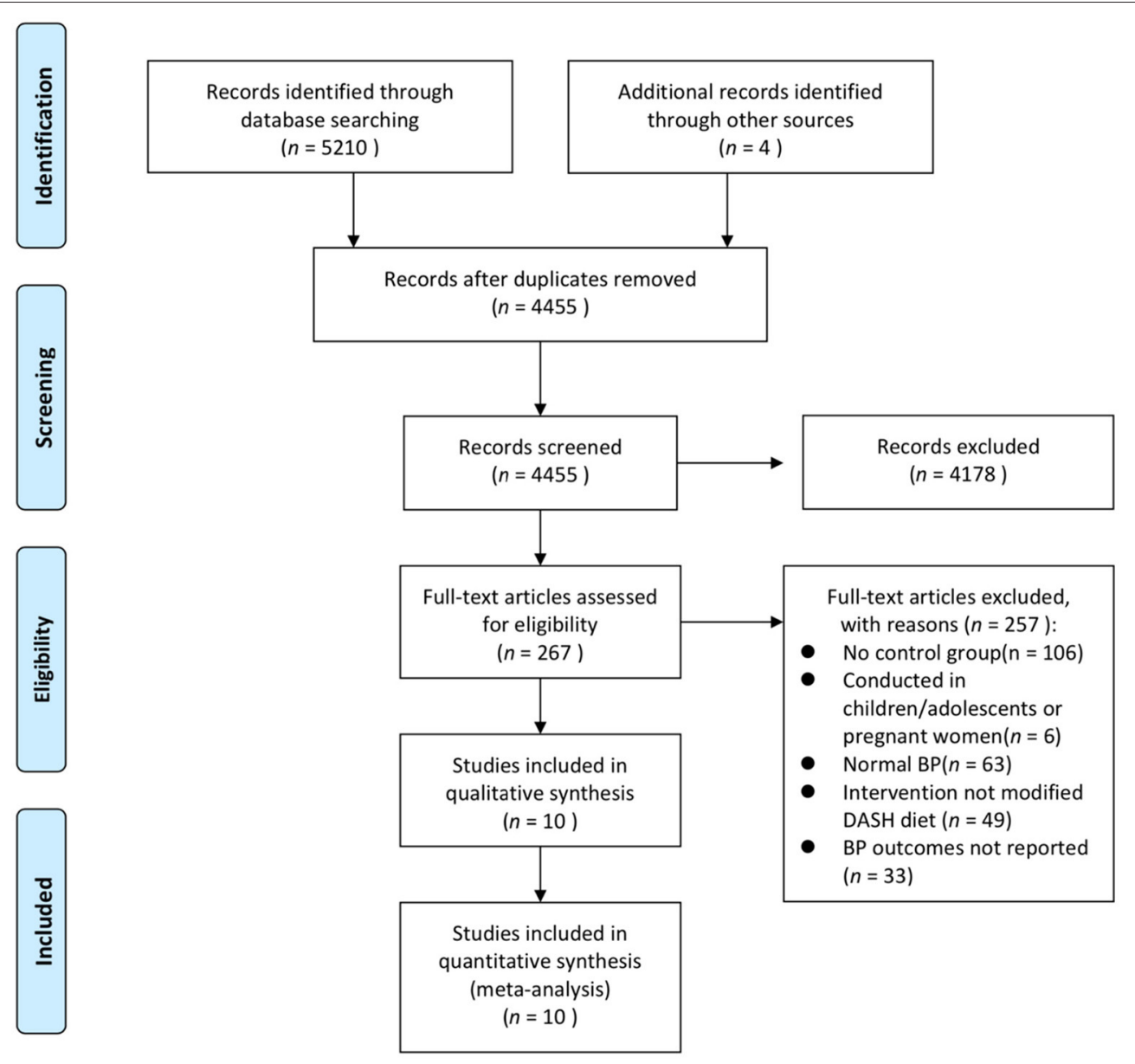

FIGURE 1 | PRISMA flowchart of the included studies. BP, blood pressure; DASH, Dietary Approaches to Stop Hypertension; RCT, randomized controlled trial.

TABLE 1 | Baseline and follow-up characteristics of included studies.

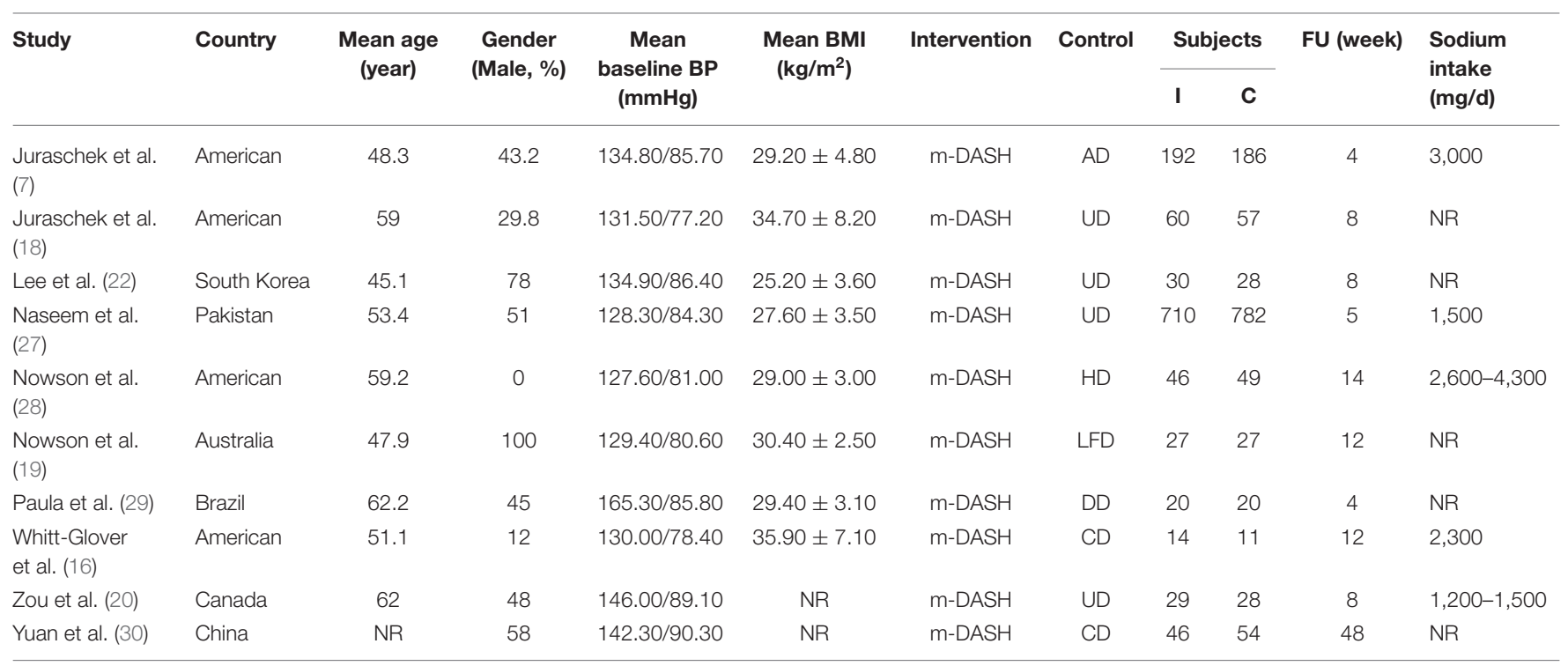

$B M I$, body mass index; BP, blood pressure; C, control; I, intervention; NR, not reported. 


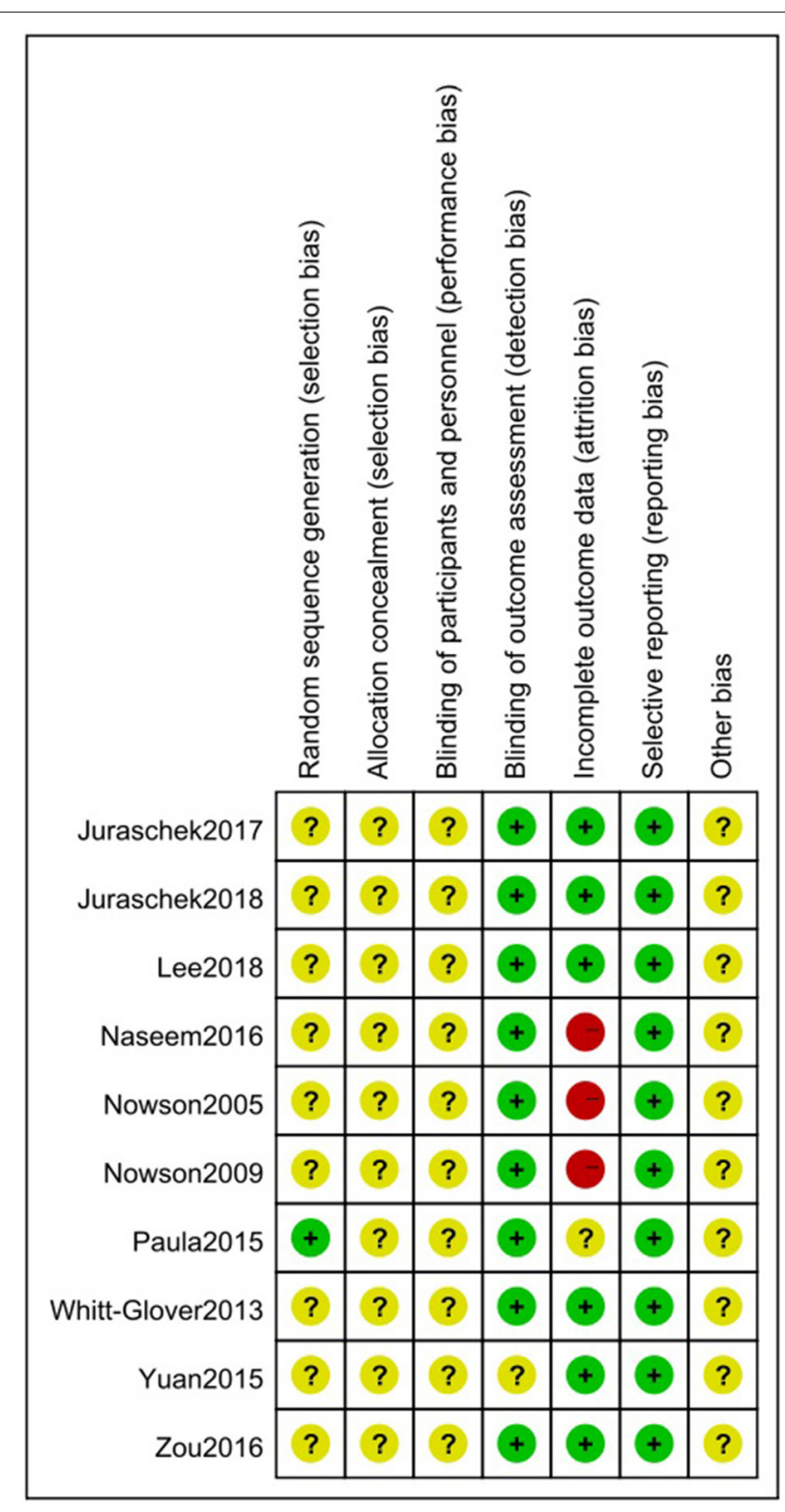

FIGURE 2 | Risk of bias summary for eligible randomized controlled trials using the Cochrane risk-of-bias tool. The symbol "+" represents a low risk of bias, the symbol "-" represents a high risk of bias, and the symbol "?" represents an unclear risk of bias.

$\mathrm{mmol} / \mathrm{L})$, or $\mathrm{HDL}(\mathrm{MD}:-1.13 \mathrm{mmol} / \mathrm{L})$ concentrations were observed between the two groups (Table 2). Additionally, no reduction in TC or HDL concentrations were observed between the two groups (Table 2).

\section{Cardiovascular Events and All-Cause Mortality}

We could not conduct a meta-analysis or systematic review of cardiovascular events and all-cause mortality because of limited data.

\section{Quality of Evidence}

Using the GRADE approach summary of evidence, the quality of evidence for the primary outcome was moderate (Supplemental Material 8).

\section{Publication Bias}

As shown by the funnel plot analysis, no publication bias was found (Figures 5, 6).

\section{Sensitivity Analysis}

Sensitivity analysis was performed to test the stability of the results by sequentially omitting one study. Our results showed that Yuan (2015) could influence the results of SBP (30).

\section{DISCUSSION}

In this study, we found that the modified DASH diet was associated with SBP and DBP reduction. Moreover, the modified DASH diet was associated with a reduction in waist circumference and TG concentration, but no statistical differences were found in weight or TC, LDL, and HDL concentrations. In subgroups of age, gender and control measures, analyses revealed that no association between the modified DASH diet and BP reduction. Although point estimates suggested that a higher daily sodium intake enhanced the BP-lowering effect, subgroup analyses did not show statistically significant differences. Furthermore, our finding indicated that DBP reduction was more prominent in groups with a more extended follow-up period or higher BMI. Moreover, SBP and DBP reduction were more pronounced in groups with a baseline SBP/DBP of $\geq 140 / 90 \mathrm{mmHg}$ compared with $<140 / 90 \mathrm{mmHg}$. The modified DASH diet might have greater positive or negative effects in combination with antihypertensive treatment or have higher accordance or provision of different interventions, such as dietary advice or food supplements, however, we did not have sufficient data for these analysis.

Like previous studies (31-33), we found that the modified DASH diet was significantly associated with reductions in SBP, DBP, and cardiovascular risk factors. However, some discrepancies in the degree of BP and cardiovascular risk factor reduction were found. We found no association between the modified DASH diet and BP reduction in subgroups stratified by sodium intake and follow-up period.

The effect of the DASH diet on BP was investigated in previous meta-analyses (10, 34-36), which were consisted with our study. However, the DASH diet reduced SBP and DBP by $4.9-7.6$ and $2.6-4.2 \mathrm{mmHg}$, respectively, which were more than our results (SBP: $3.26 \mathrm{mmHg}$; DBP: $2.07 \mathrm{mmHg}$ ). The reasons might be that we contained pre-hypertensive patients. In addition, subgroup analyses found that the mean BP reduction (SBP: $7.59 \mathrm{mmHg}$; DBP: $4.47 \mathrm{mmHg}$ ) in patients with a baseline SBP/DBP threshold of $\geq 140 / 90 \mathrm{mmHg}$ was similar to the BP range in the previous studies. Furthermore, Filippou et al. (11) found that in adults with and without 


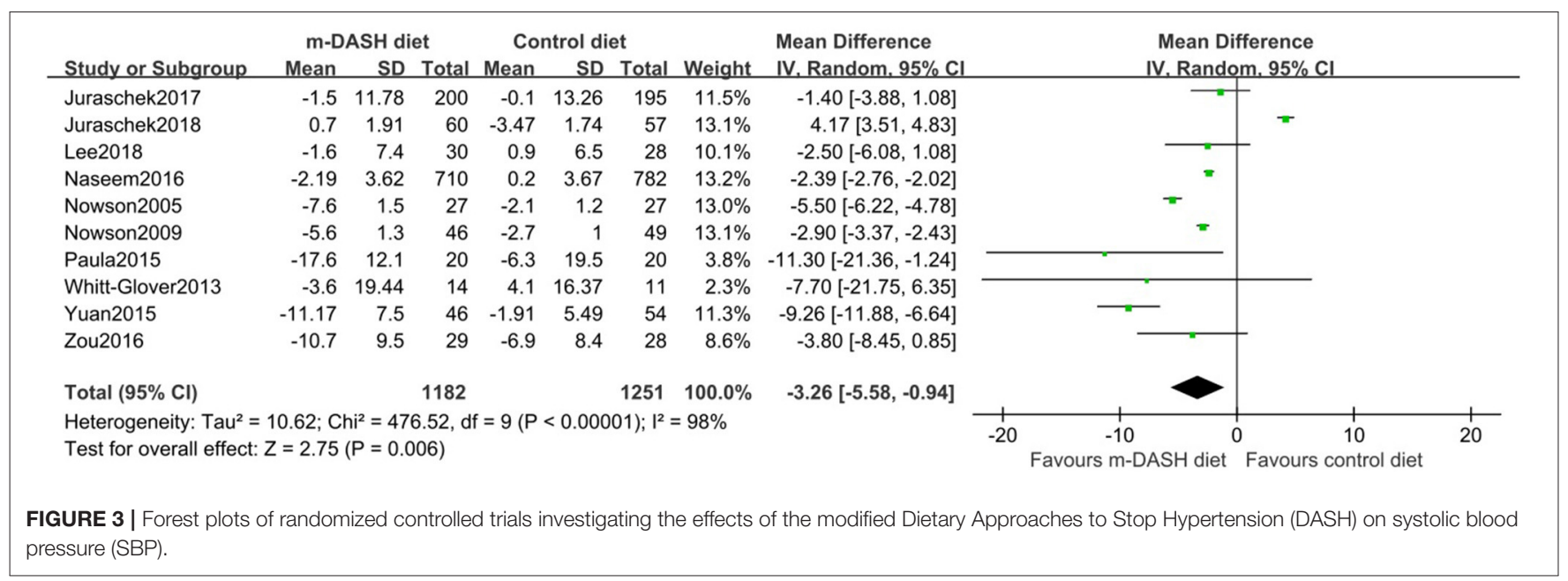

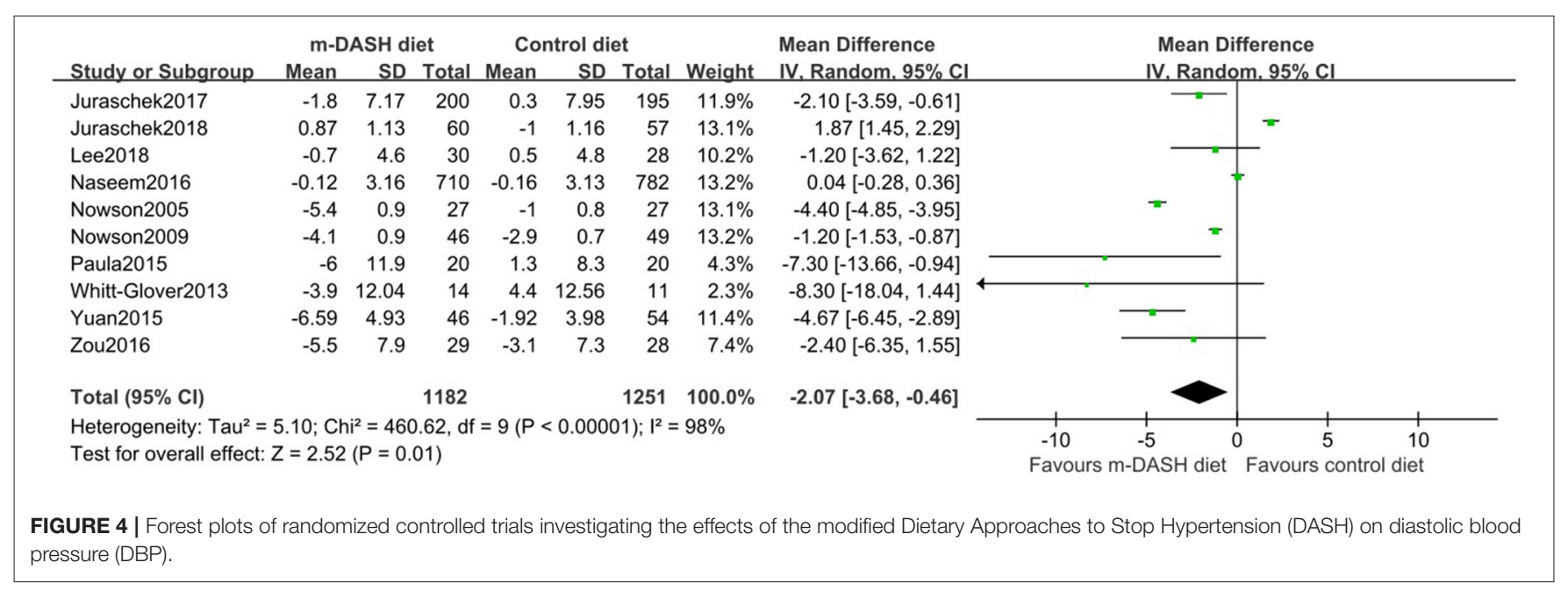

TABLE 2 | Effect of the modified DASH diet in adults with increased BP for cardiovascular risk factors.

\begin{tabular}{lccccc}
\hline Outcome & $\mathbf{R C T}(\boldsymbol{n})$ & Subjects $(\boldsymbol{n})$ & SMD $(\mathbf{9 5 \%} \mathbf{c l})$ & \multirow{2}{*}{$\%$} & $\boldsymbol{P}$ \\
\hline Weight & 2 & 112 & $-0.31[-0.61,-0.01]$ & 0 & 0.05 \\
Waist & 2 & 98 & $-1.57[-2.98,-0.15]$ & 0 & 0.03 \\
TC & 3 & 1,586 & $-0.98[-2.46,0.50]$ & 96 & 0.19 \\
TG & 2 & 94 & $-1.04[-1.47,-0.60]$ & 7 & 0.00001 \\
LDL & 3 & 1,586 & $-0.41[-1.06,0.24]$ & 85 & 0.21 \\
HDL & 2 & 1,546 & $-1.13[-3.41,1.14]$ & 98 & 0.33 \\
\hline
\end{tabular}

BP, blood pressure; DASH, Dietary Approaches to Stop Hypertension; RCT, randomized controlled trial; TC, total cholesterol; TG, triglyceride; LDL, low-density lipoprotein; HDL, high-density lipoprotein.

hypertension, the DASH diet could reduce SBP and DBP by 3.2 and $2.5 \mathrm{mmHg}$, respectively, which was similar to our study.

Wong et al. conducted a RCT at primary care institutions and showed that as follow-up time was extended, patients' dietary compliance and BP reduction decreased (37), which

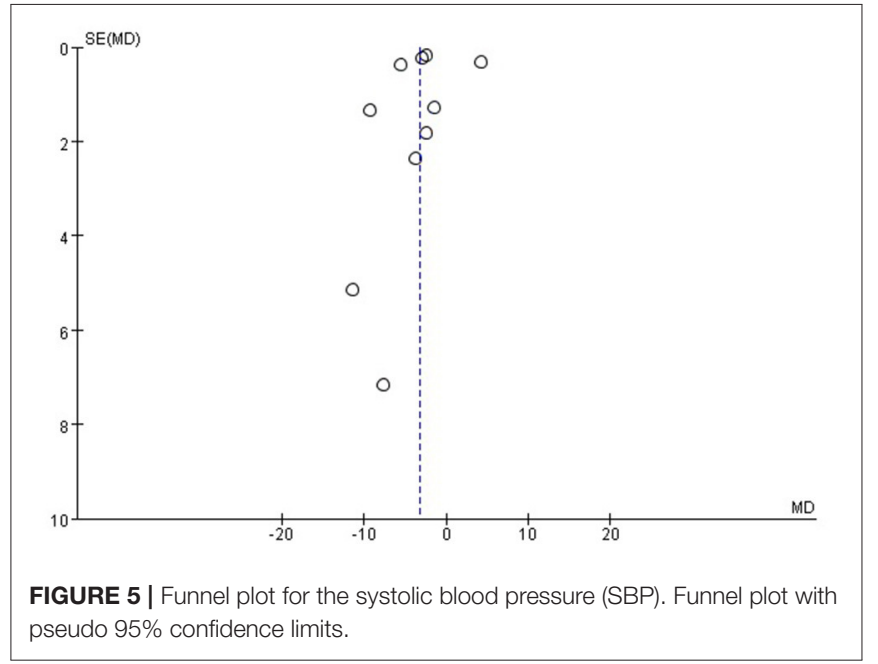

contradicted with our study findings. Most studies included in our study had a follow-up period of 4-12 weeks, which might have been too short to observe a decrease in dietary 


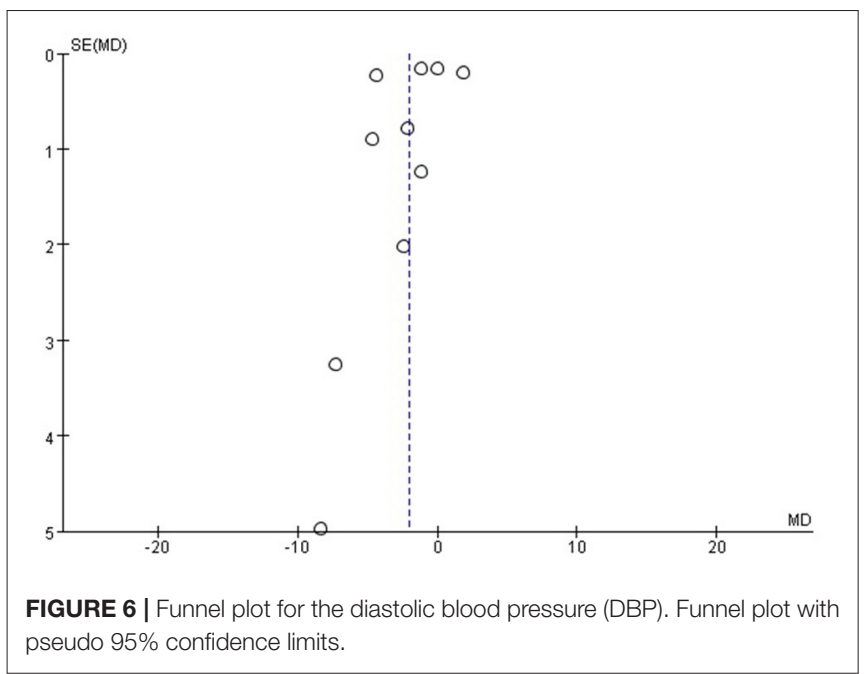

compliance. Thus, more studies with a longer follow-up time were still needed.

A study showed that more than $30 \%$ of hypertension cases were caused by excessive salt intake (38); thus, BP decreased with sodium intake decreased (33). However, contradictory to other studies (31), we found a lower sodium supplication could not lead to better BP reduction. This might because the modified DASH diet was strict sodium restriction, which was generally as low as $3,000 \mathrm{mg}$. That also suggested that too low sodium intake did not reduce BP further but might reduce adherence to dietary treatment.

The resutls of meta-regression showed that control measures (healthy diet or common diet) appeared to be the main source of heterogeneity for SBP and DBP. However, in our subgroup analysis, no matter compared with healthy diet or common diet, the modified DASH diet was associated with a significant reduction in SBP and DBP, and no statistical difference was observed in the two subgroups. Thus, even in the presence of heterogeneity, we could still conclude that the modified DASH diet could reduce $\mathrm{BP}$.

A previous meta-analysis showed that the DASH diet was associated with an increased HDL concentration (17), and a reduction in waist circumference $(32), \operatorname{BMI}(31,32)$, and TG, TC, and LDL concentrations (17). That was different from our findings, which did not show a reduction in waist circumference or TG concentration, and might be a consequence of the small sample size.

It was suggested that DASH diet could reduce $\mathrm{BP}$ and alleviate cardiovascular disease $(2,17,39-42)$. Our study showed that the modified DASH diet had similar effects on BP reduction when compared with the DASH diet. Meanwhile, the modified DASH diet improved dietary adherence by enhancing the feasibility and acceptability of the diet. Hence, the modified DASH diet was an effective treatment for hypertensive patients.

There were some limitations to the study. First, some studies did not provide sufficient information about study design or other details. Second, the overall heterogeneity among studies was high and was partly downgraded by explanatory analyses. Third, although subgroup analyses were conducted across mean clinical thresholds in each separate trial, it was undetermined individual trial patients were above or below these preselected thresholds. Finally, the included study did not report or described the blinding process and methods in detail, thus no further analysis was performed.

\section{CONCLUSION}

In conclusion, the current systematic review and metaanalysis demonstrates that a modified DASH diet can reduce $\mathrm{BP}$ in pre-hypertensive and hypertensive patients. A higher baseline BP is associated with more prominent systolic and diastolic BP reduction. Further largesample and high-quality studies are needed to verify the above conclusions.

\section{DATA AVAILABILITY STATEMENT}

The original contributions presented in the study are included in the article/Supplementary Files, further inquiries can be directed to the corresponding authors.

\section{AUTHOR CONTRIBUTIONS}

YL and Y-GZ designed the study and edited the manuscript. RG, RY, X-YL, YZ, B-FZ, QZ, and LC searched the data, analyzed the data, drafted the manuscript. NL revised the manuscript. All authors approved the final version of the manuscript.

\section{FUNDING}

This study was supported by the grant of the National Key Research and Development Program of China (No. 2017YFC0907303); Science \& Technology Department of Sichuan province (No. 2020YFSY0014 and 2020YFS0186); National Clinical Research Center for Geriatrics, West China Hospital, Sichuan University (Z20191009).

\section{ACKNOWLEDGMENTS}

We thank Emily Woodhouse, Ph.D., from Liwen Bianji, Edanz Editing China (www.liwenbianji.cn/ac), for editing the English text of a draft of this manuscript.

\section{SUPPLEMENTARY MATERIAL}

The Supplementary Material for this article can be found online at: https://www.frontiersin.org/articles/10.3389/fnut.2021. 725020/full\#supplementary-material 


\section{REFERENCES}

1. Global Health Observatory (GHO) data: raised blood pressure: situation and trends. World Health Organization (2020). Available online at: http://www. who.int/gho/ncd/risk_factors/blood_pressure_prevalence_text/en/ (accessed: July 1, 2021).

2. Sotos-Prieto M, Bhupathiraju SN, Mattei J, Fung TT, Li Y, Pan A, et al. Association of changes in diet quality with total and cause-specific mortality. N Engl J Med. (2017) 377:143-53. doi: 10.1056/NEJMoa1613502

3. Steinberg D, Bennett GG, Svetkey L. The DASH diet, 20 years later. JAMA. (2017) 317:1529-30. doi: 10.1001/jama.2017.1628

4. Lewington S, MacMahon S, Peto R, Aromaa A, Baigent C, Carstensen J, et al. Age-specific relevance of usual blood pressure to vascular mortality: a metaanalysis of individual data for one million adults in 61 prospective studies. Lancet. (2002) 360:1903-13. doi: 10.1016/s0140-6736(02)11911-8

5. Lawes CM, Rodgers A, Bennett DA, Parag V, Suh I, Ueshima H, et al. Blood pressure and cardiovascular disease in the Asia Pacific region. J Hypertens. (2003) 21:707-16. doi: 10.1097/00004872-200304000-00013

6. Appel LJ, Champagne CM, Harsha DW, Cooper LS, Obarzanek E, Elmer PJ, et al. Effects of comprehensive lifestyle modification on blood pressure control main results of the PREMIER clinical trial. JAMA. (2003) 289:2083-93. doi: 10.1001/jama.289.16.2083

7. Juraschek SP, Woodward M, Sacks FM, Carey VJ, Miller ER III, Appel LJ. Time course of change in blood pressure from sodium reduction and the DASH diet. Hypertension. (2017) 70:923-9. doi: 10.1161/HYPERTENSIONAHA.117.10017

8. Tyson CC, Nwankwo C, Lin PH, Svetkey LP. The Dietary approaches to stop hypertension (DASH) eating pattern in special populations. Curr Hypertens Rep. (2012) 14:388-96. doi: 10.1007/s11906-012-0296-1

9. Appel LJ, Moore TJ, Obarzanek E, Vollmer WM, Svetkey LP, Sacks FM, et al. A clinical trial of the effects of dietary patterns on blood pressure. DASH collaborative research group. N Engl J Med. (1997) 336:1117-24. doi: 10.1056/NEJM199704173361601

10. Siervo M, Lara J, Chowdhury S, Ashor A, Oggioni C, Mathers JC. Effects of the Dietary Approach to stop hypertension (DASH) diet on cardiovascular risk factors: a systematic review and meta-analysis. Br J Nutr. (2015) 113:1-15. doi: 10.1017/S0007114514003341

11. Filippou CD, Tsioufis CP, Thomopoulos CG, Mihas CC, Dimitriadis KS, Sotiropoulou LI, et al. Dietary approaches to stop hypertension (DASH) diet and blood pressure reduction in adults with and without hypertension: a systematic review and meta-analysis of randomized controlled trials. $A d v$ Nutr. (2020) 11:1150-60. doi: 10.1093/advances/nmaa041

12. Williams B, Mancia G, Spiering W, Agabiti Rosei E, Azizi M, Burnier M, et al. $2018 \mathrm{ESC} / \mathrm{ESH}$ Guidelines for the management of arterial hypertension: the task force for the management of arterial hypertension of the European Society of Cardiology and the European Society of Hypertension: the task force for the management of arterial hypertension of the European Society of Cardiology and the European Society of Hypertension. J Hypertens. (2018) 36:1953-2041. doi: 10.1097/HJH.0000000000001940

13. Unger T, Borghi C, Charchar F, Khan NA, Poulter NR, Prabhakaran D, et al. 2020 International society of hypertension global hypertension practice guidlines journal of Hypertension. Hypertension. (2020) 75:982-1004. doi: 10.1097/HJH.0000000000002453

14. Chiu S, Bergeron N, Williams PT, Bray GA, Sutherland B, Krauss RM. Comparison of the DASH (Dietary approaches to stop hypertension) diet and a higher-fat DASH diet on blood pressure and lipids and lipoproteins: a randomized controlled trial1-3. Am J Clin Nutr. (2016) 103:341-7. doi: $10.3945 /$ ajcn.115.123281

15. Nakamura M, Ojima T. A modified DASH diet is one possible solution for overcoming the unfavorable link between vegetable and salt intake in the Japanese diet. Hypertens Res. (2016) 39:756-7. doi: 10.1038/hr.2016.83

16. Whitt-Glover MC, Hunter JC, Foy CG, Quandt SA, Vitolins MZ, Leng I, et al. Translating the dietary approaches to stop hypertension (DASH) diet for use in underresourced, urban African American communities, 2010. Prev Chronic Dis. (2013) 10:120088. doi: 10.5888/pcd10.120088

17. Tiong XT, Nursara Shahirah A, Pun VC, Wong KY, Fong AYY, Sy RG, et al. The association of the dietary approach to stop hypertension (DASH) diet with blood pressure, glucose and lipid profiles in Malaysian and
Philippines populations. Nutr Metab Cardiovasc Dis. (2018) 28:856-63. doi: 10.1016/j.numecd.2018.04.014

18. Juraschek SP, White K, Tang O, Yeh H-C, Cooper LA, Miller ER III. Effects of a dietary approach to stop hypertension (DASH) diet intervention on serum uric acid in African Americans with hypertension. Arthritis Care Res. (2018) 70:1509-16. doi: 10.1002/acr.23515

19. Nowson CA, Worsley A, Margerison C, Jorna MK, Godfrey SJ, Booth A. Blood pressure change with weight loss is affected by diet type in men. Am J Clin Nutr. (2005) 81:983-9. doi: 10.1093/ajcn/81.5.983

20. Zou P, Dennis C-L, Lee R, Parry M. Dietary approach to stop hypertension with sodium reduction for Chinese Canadians (DASHNa-CC): a pilot randomized controlled trial. J Nutr Health Aging. (2016) 21:1225-32. doi: 10.1007/s12603-016-0861-4

21. Kim H, Song H-J, Han H-R, Kim KB, Kim MT. Translation and validation of the dietary approaches to stop hypertension for Koreans intervention culturally tailored dietary guidelines for Korean Americans with high blood pressure. J Cardiovasc Nurs. (2013) 28:514-23. doi: 10.1097/JCN.0b013e318262c0c1

22. Lee CJ, Kim JY, Shim E, Hong SH, Lee M, Jeon JY, et al. The effects of diet alone or in combination with exercise in patients with prehypertension and hypertension: a randomized controlled trial. Korean Circ J. (2018) 48:637-51. doi: $10.4070 / \mathrm{kcj} .2017 .0349$

23. Gajewska D, Kucharska A, Kozak M, Wunderlich S. Effectiveness of individual nutrition education compared to group education, in improving anthropometric and biochemical indices among hypertensive adults with excessive body weight: a randomized controlled trial. Nutrients. (2019) 11:2921. doi: $10.3390 /$ nu11122921

24. Yao X, Florez ID, Zhang P, Zhang C, Zhang Y, Wang C, et al. Clinical research methods for treatment, diagnosis, prognosis,etiology, screening, and prevention: a narrative review. J Evid Based Med. (2020) 13:130-6. doi: $10.1111 /$ jebm. 12384

25. Cochrane Handbook for Systematic Reviews of Interventions version 6.0. Cochrane (2019). Available online at: www.training.cochrane.org/handbook (accessed: July 1, 2021).

26. Review Manager (RevMan) Version 5.4.1. The Cochrane Collaboration (2020). Available online at: https://training.cochrane.org/online-learning/ core-software-cochrane-reviews/revman/revman-5-download/downloadand-installation (accessed: July 1, 2021).

27. Naseem S, Ghazanfar H, Assad S, Ghazanfar A. Role of sodium-restricted dietary approaches to control blood pressure in Pakistani hypertensive population. J Pak Med Assoc. (2016) 66:837-42.

28. Nowson CA, Wattanapenpaiboon N, Pachett A. Low-sodium dietary approaches to stop hypertension-type diet including lean red meat lowers blood pressure in postmenopausal women. Nutr Res. (2009) 29:8-18. doi: 10.1016/j.nutres.2008.12.002

29. Paula TP, Viana LV, Neto ATZ, Leitao CB, Gross JL, Azevedo MJ. Effects of the DASH diet and walking on blood pressure in patients with Type 2 diabetes and uncontrolled hypertension: a randomized controlled trial. J Clin Hypertens. (2015) 17:895-901. doi: 10.1111/jch.12597

30. Yuan Q, Zhang XJ, Xie Y, Zhao L, Zhang YL. Effect of modified DASH diet on blood pressure and quality of life in patients with essential hypertension. Pract Prev Med. (2015) 22: 1055-7. doi: 10.1056/NEJM2001010434 40101

31. Steinberg D, Kay M, Burroughs J, Svetkey LP, Bennett GG. The effect of a digital behavioral weight loss intervention on adherence to the Dietary Approaches to Stop Hypertension (DASH) dietary pattern in medically vulnerable primary care patients: results from a randomized controlled trial. $J$ Acad Nutr Diet. (2019) 119:574-84. doi: 10.1016/j.jand.2018.12.011

32. Kucharska A, Gajewska D, Kiedrowski M, Sińska B, Juszczyk G, Czerw A, et al. The impact of individualised nutritional therapy according to DASH diet on blood pressure, body mass, and selected biochemical parameters in overweight/obese patients with primary arterial hypertension: a prospective randomised study. Kardiol Pol. (2018) 76:158-65. doi: 10.5603/KP.a2017.0184

33. Najafi A, Faghih S, Hojhabrimanesh A, Najafi M, Tangestani H, Atefi M, et al. Greater adherence to the dietary approaches to stop hypertension (DASH) dietary pattern is associated with lower blood pressure in healthy Iranian primary school children. Eur J Nutr. (2018) 57:1449-58. doi: $10.1007 / \mathrm{s} 00394-017-1423-1$ 
34. Saneeiv P, Salehi-Abargouei A, Esmaillzadeh A, Azadbakht L. Influence of dietary approaches to stop hypertension (DASH) diet on blood pressure: a systematic review and meta-analysis on randomized controlled trials. Nutr Metab Cardiovasc Dis. (2014) 24:1253-61. doi: 10.1016/j.numecd.2014.06.008

35. Ndanuko RN, Tapsell LC, Charlton KE, Neale EP, Batterham MJ. Dietary patterns and blood pressure in adults: a systematic review and metaanalysis of randomized controlled trials. Adv Nutr. (2016) 7:76-89. doi: 10.3945/an.115.009753

36. Gay HC, Rao SG, Vaccarino V, Ali MK. Effects of different dietary interventions on blood pressure: systematic review and meta-analysis of randomized controlled trials. Hypertension. (2016) 67:733-9. doi: 10.1161/HYPERTENSIONAHA.115.06853

37. Wong MCS, Wang HHX, Kwan MWM, Li STS, Liang M, Fung FDH, et al. The effectiveness of dietary approaches to stop hypertension (DASH) counselling on estimated 10-year cardiovascular risk among patients with newly diagnosed grade 1 hypertension: a randomised clinical trial. Int $J$ Cardiol. (2016) 224:79-87. doi: 10.1016/j.ijcard.2016.08.334

38. Shah NS, Leonard D, Finley CE, Rodriguez F, Sarraju A, Barlow $\mathrm{CE}$, et al. Dietary patterns and long-term survival: a retrospective study of healthy primary care patients. Am J Med. (2018) 131:48-55. doi: 10.1016/j.amjmed.2017.08.010

39. Campos CL, Wood A, Burke GL, Bahrami H, Bertoni AG. Dietary approaches to stop hypertension diet concordance and incident heart failure: the multi-ethnic study of atherosclerosis. Am J Prev Med. (2019) 56:819-26. doi: 10.1016/j.amepre.2018.11.022

40. Talaei M, Koh WP, Yuan JM, van Dam RM. DASH dietary pattern, mediation by mineral intakes, and the risk of coronary artery disease and stroke mortality. J Am Heart Assoc. (2019) 8:e011054. doi: 10.1161/JAHA.118.011054
41. Jones NRV, Forouhi NG, Khaw KT, Wareham NJ, Monsivais P. Accordance to the dietary approaches to stop hypertension diet pattern and cardiovascular disease in a British, population-based cohort. Eur J Epidemiol. (2018) 33:23544. doi: 10.1007/s10654-017-0354-8

42. Soltani S, Arablou T, Jayedi A, Salehi-Abargouei A. Adherence to the dietary approaches to stop hypertension (DASH) diet in relation to all-cause and cause-specific mortality: a systematic review and doseresponse meta-analysis of prospective cohort studies. Nutr J. (2020) 19:37. doi: 10.1186/s12937-020-00554-8

Conflict of Interest: The authors declare that the research was conducted in the absence of any commercial or financial relationships that could be construed as a potential conflict of interest.

Publisher's Note: All claims expressed in this article are solely those of the authors and do not necessarily represent those of their affiliated organizations, or those of the publisher, the editors and the reviewers. Any product that may be evaluated in this article, or claim that may be made by its manufacturer, is not guaranteed or endorsed by the publisher.

Copyright (c) 2021 Guo, Li, Yang, Liao, Zhang, Zhu, Zhao, Chen, Zhang and Lei. This is an open-access article distributed under the terms of the Creative Commons Attribution License (CC BY). The use, distribution or reproduction in other forums is permitted, provided the original author(s) and the copyright owner(s) are credited and that the original publication in this journal is cited, in accordance with accepted academic practice. No use, distribution or reproduction is permitted which does not comply with these terms. 